\title{
RELACIONES VITALES: EL AULA COMO ESCENARIO PERMANENTE DE INVESTIGACIÓN
}

Luz Elena Toro González

Departamento de Estudios

Educativos

luzelena.toro@ucaldas.edu.co

Artículo de Reflexión

Recepción: 26 de mayo de 2014 Aprobación: 30 de julio de 2014

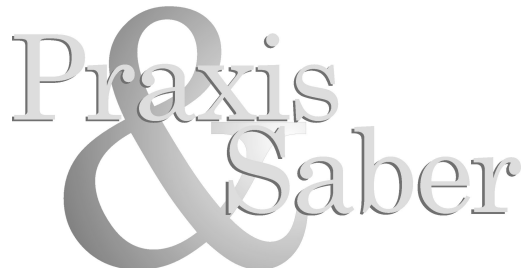

Revista de Investigación y Pedagogía Maestría en Educación. Uptc

\section{Resumen}

El documento plantea el potencial de la investigación en la transformación del sujeto y su conocimiento: para ello encuentra en la educación una posibilidad de crecer y de ser reconocida desde diversas categorías en tanto estrategia formativa y como herramienta necesaria en la responsabilidad del profesional con su disciplina, así como con la generación de conocimiento. A partir de allí se analizan los elementos que le son propios al docente en su quehacer cotidiano, definiendo así: (a) la pedagogía como guía en la formación del ser humano; (b) la didáctica como las estrategias que se diseñan para la relación ecuánime entre enseñanza y aprendizaje, afianzando la relación maestroestudiante; (c) el currículo como la organización, priorización y análisis de saberes que fluyen en el aula; y (d) la evaluación como elemento valorativo de todo el proceso para su resignificación. Finalmente se expone la conjunción de dichos elementos con los propósitos estructurales de la investigación para generar una propuesta, de tantas posibles, que apoye al maestro en la decisión de investigar sin que ello implique un gasto innecesario de tiempo y recursos a su disposición.

Palabras clave: educación, formación, investigación, maestro, transformación. 


\title{
Vital Relationships: ClassRoOM AS AN OngOING RESEARCH SETTING
}

\begin{abstract}
This paper considers the potential research has in transforming subjects and their knowledge, and for this, it finds in education a possibility of growing and being acknowledged, from diverse categories, as a formative strategy and as a tool required in the responsibility a professional has with both his/her discipline and the knowledge generation. Based on this, the characteristic elements of a teacher's day-to-day work are analyzed; defining in this way (a) Pedagogy as a guide in human being formation; (b) Didactics as a set of strategies designed for an impartial relationship between teaching and learning, strengthening the studentteacher relationship; (c) Curricula as the organization, prioritization and analysis of the knowledge flowing in the classroom; and (d) Evaluation as an assessment element of the whole process to redefine it. Finally, the conjunction of these elements with the research structural aims is explained to generate one of many proposals, that supports teachers in their decision to research, which does not mean wasting time or available resources.
\end{abstract}

Keywords: education, formation, research, teacher, transformation.

\section{Relations Vitales: La Salle de Classe Comme Cadre Permanent de Recherche}

\section{Résumé}

Le document présente le potentiel de la recherche dans la transformation du sujet et sa connaissance; pour ce faire, il trouve dans l'éducation une possibilité de croître et d'être reconnu selon diverses catégories en tant que stratégie formative et comme outil nécessaire dans la responsabilité du professionnel avec sa discipline, ainsi que la création de connaissances. Partant de là, les éléments propres à l'enseignant dans ses tâches quotidiennes sont analysés, en définissant: (a) la pédagogie comme étant 
le guide dans la formation de l'être humain; (b) la didactique comme les stratégies créées pour la relation équitable entre l'enseignement et l'apprentissage, en renforçant la relation maitre-étudiant; (c) le cursus en tant qu'organisation, priorisation et analyse des savoirs qui découlent dans la salle de classe; et (d) l' évaluation comme élément de mesure de tout le processus pour sa resignification. Finalement la conjonction de ces éléments est exposée dans des buts structuraux de recherche afin de créer une proposition, parmi plusieurs possibles, qui puisse aider le maitre dans sa décision de recherche sans que cela implique une perte de temps inutile mettant les ressources à sa disposition.

Mots clés: éducation, formation, recherche, maitre, transformation.

\section{Relações Vitais: A Sala de Aula Como Cenário Permanente de Pesquisa}

\section{Resumo}

O texto explana o potencial da pesquisa na transformação do sujeito e seu conhecimento; para isto encontra na educação uma possibilidade de crescer e de ser reconhecida desde diversas categorias como estratégia formativa e como instrumento necessário na responsabilidade do profissional com a sua disciplina, assim como com a geração de conhecimento. A partir de aí se analisam os elementos que lhe são próprios ao docente em seu fazer diário, definindo assim: (a) a pedagogia como guia na formação do ser humano; (b) a didática como as estratégias que se delineiam para a relação equitativa entre ensino e aprendizagem, consolidando a relação maestroaluno; (c) o currículo como a organização, priorização e análise de saberes que nascem na sala de aula; e (d) a avaliação como elemento valorativo de todo o processo para a sua resignificação. Finalmente se expõe a conjunção de ditos elementos com os propósitos estruturais da pesquisa para gerar uma proposta, de tantas possíveis, que ajude ao professor na decisão de pesquisar sem que isto implique um gasto desnecessário de tempo e recursos a sua disposição.

Palavras chave: educação, formação, pesquisa, professor, transformação. 


\section{Introducción}

La complejidad de las situaciones sociales, locales y globales exige, para la formación permanente del ser humano, otras miradas desde la relación maestro - estudiante que permitan la deconstrucción y reconstrucción del saber, teniendo como excusa el conocimiento que fluye entre unos y otros; así la escuela se renueva y recobra para sí su valor como templo del saber.

No obstante, para esto es de vital importancia la producción y transformación de conocimiento por parte del docente, en un proceso que transversalice el análisis de contexto y la redefinición de objetivos con proyección al cambio social, actualización e innovación de saberes y estrategias de intervención; además, la valoración del estudiante y la dinámica educativa.

En este sentido la presencia de un maestro investigador, invita a vivir y comprender la escuela y sus dinámicas como escenario propicio para lograr aquello que otrora se consideraría utópico, pues la utopía no es lo imposible, sino la frontera de la creación y, está en la investigación, una de las oportunidades más acertadas para lograrlo; tal como lo menciona Botero Uribe (s.f.) al referirse a la investigación como proceso creativo:

El pensar ya implica una perspectiva utopistica. El pensar es una ventana a lo concebible, la búsqueda de nuevas formas, de posibilidades no exploradas. El pensar otea lo desconocido, aquello, que sin saber muy bien por qué desde el comienzo, puede ayudarnos a resolver muchos problemas. [...] La Utopía es la posibilidad de formular propuestas abiertas, que pueden reformularse, adaptarse, mejorarse e incluso sustituirse cuando no sirvan (1).

A partir de lo anterior se concibe la presente apuesta en acciones conjuntas entre investigación y educación, en la solidez de un proceso de formación que involucre la imaginación, la creación y la transformación de la realidad social y educativa.

\section{La investigación como apertura}

En la actualidad pensar la investigación es aproximarse a la incertidumbre, a la curiosidad, a ese deseo incontenible de ir en busca de lo incierto, es 
quizás revivir aquel ideal socrático de alcanzar la felicidad a través del saber, de la mayéutica, es decir, mediante el cuestionamiento permanente acerca del mundo natural y social que nos rodea; pero embarcarse en este recorrido de descubrimiento no está desprovisto de organización, no es al azar y mucho menos es una simple indagación. A este respecto Bedoya (2005) plantea la investigación como un "[...] proceso que tiene como objetivo producir ciencia, buscar la explicación de la realidad mediante un método riguroso [...]” (138)

Investigar es, en sí misma, una acción planeada y organizada que el sujeto-investigador emprende para resolver una duda, podríamos decir, disciplinar ${ }^{1}$. Pero para ello se debe tener claridad sobre su punto de partida, de la pertinencia de la investigación en términos de que no se haya resuelto o abordado a través de otros estudios. Este tipo de acciones investigativas se pueden comprender en el marco de los motivos porque que plantea Schutz (2008): “[...] los motivos a los que se explica sobre la base de los antecedentes, ambiente o predisposición psíquica del actor $[\ldots]$ están dominados por el pasado [...] forman una categoría objetiva" (26).

Estos motivos se refieren entonces, por un lado, al contexto que rodea la naturaleza de la investigación, las razones que la componen y la hacen un asunto necesario de ser investigado para llenar los vacíos en el conocimiento disciplinar; en términos de la investigación, se refieren a aquellos elementos que permiten plantear el problema de investigación y generar una pregunta.

De igual manera es completamente necesario definir el horizonte al cual queremos llegar, si bien la investigación es una búsqueda, como ya se ha mencionado, el investigador debe diseñar algunos objetivos o metas a cumplir que les guíe, como un faro hacia el cual dirigirnos; retomando a Schutz (2008), este autor se refiere a los motivos para como "Los motivos que implican fines a lograr, objetivos que se procura alcanzar [...] están dominados por el tiempo futuro [...] forman una categoría subjetiva" (26).

1 Al respecto Hernández y López (2002) plantean que: “Aunque la palabra disciplina señale en primera instancia una región del conocimiento, sugiere al mismo tiempo un campo de trabajo y de relación social a los cuales se accede a través de un proceso de formación caracterizado por la importancia que se reconoce al ejercicio de la investigación" (12). 
Definida la naturaleza de la investigación y el horizonte al cual llegar, es necesario planear el recorrido, construir la carta de navegación que determinará paso a paso el camino que el investigador deberá transitar en la búsqueda de saciar su deseo de conocimiento y de encontrar las respuestas a sus inquietudes. Así, la metodología es el diseño que da respuesta al "cómo" alcanzar los objetivos que permitirán dar respuesta a las preguntas planteadas por el investigador.

No obstante, si bien como la definen Hernández, Fernández y Baptista (2010) "La investigación es un conjunto de procesos sistemáticos, críticos y empiricos que se aplican al estudio de un fenómeno" (4), es necesario comprender que en la actualidad es difícil continuar con las pretensiones de exactitud que la ciencia exige desde hace más de dos siglos. Esto debido a lo transitorio de los conocimientos producidos por la investigación, a la era de las tecnologías y las comunicaciones donde los saberes emergen día tras día y unos tras otros, sin dar tiempo al ser humano para que los apropie, los utilice, y luego los transforme.

En ese sentido se plantea la necesidad de que los métodos de investigación, principalmente los que son propios de las ciencias humanas y sociales (Dos Santos \& Sánchez, 1997), se adapten constantemente a estos cambios, y en esa medida es imposible asumirlos de la manera rigurosa como fueron concebidos originalmente; es por ello que hoy por hoy encontramos múltiples variaciones sobre dichos métodos, refiriéndonos ya no sólo - por ejemplo- a la etnografía o la fenomenología, sino a las etnografías y fenomenologías, en plural, pues la diversidad de fenómenos que estudian y los sujetos inmersos en ellos, han exigido flexibilizarlos, adaptarlos y transformarlos para que su aproximación a la realidad sea más pertinente.

De igual manera otros autores de la investigación han expuesto diseños emergentes que proponen una suerte de collage entre los métodos de investigación atendiendo a la complejidad propia de los grupos sociales, planteando una complementariedad epistemológica y metodológica en los procesos investigativos (Murcia \& Jaramillo, 2001 y 2008).

Estos tres elementos en conjunto, problema (¿por qué?), objetivos (¿para qué?) y metodología (¿cómo?), definen la coherencia interna de cualquier 
proceso investigativo, sin los cuales ésta no podría ser, entendida como la relación íntima entre los principios, los fines y el camino para llegar del primero al segundo; esta coherencia determina la validez, pertinencia y fiabilidad de los hallazgos arrojados durante la aplicación de las técnicas utilizadas durante el estudio.

\section{Investigación y educación}

[...] el educar y el investigar, apenas metáforas de espirales infinitamente ascendentes y de realidades supremamente complejas, sugieren tránsitos que van y vuelven desde las cosas mismas a su más intrincados contextos, búsquedas al fin y al cabo interminables e incesantes que procuran edificar y rehacer sus sentidos en cada espacio vital, en toda práctica social y acto de lenguaje; en pensamiento y sensibilidades desencadenados que así como unas veces dan sustento y validez a renovadas relaciones de sentido, también en otras y como irreductible vocación de ruptura, desde la radicalidad humana del acto de preguntar y de la pasión por lo aparentemente obvio, se oponen a toda pretensión de certeza incontrovertible, a toda tentación de un punto final para las aventuras del pensar y del sentir (Gutiérrez Ruiz en Deslauriers, 2004: XXI).

Para Bedoya (2005) la investigación debe dirigirse hacia la formación del sujeto $^{2}$ (tanto del docente como del estudiante); en este sentido, construir la relación entre investigación y educación necesariamente nos lanza por dos vertientes, que a su vez se subdividen como se expone a continuación (ilustración 1):

2 "Investigar en educación debe tener esta orientación o sentido fundamental: se investiga no tanto para producir conocimiento nuevo con el que se pretendería llegar a la explicación definitiva del problema (o de los datos de los que se habría partido) sino para que a través de este proceso llamado investigación (o proceso de conocimiento) se dé o se afiance mejor el proceso de formación (concebido como un proceso integral en el que no sólo el adelanto científico - o conocer cada vez más - está presente, sino la construcción de la subjetividad como entidad en capacidad que está de serlo)" (Bedoya, 2005: 141). 
Ilustración 1. Formas de relación entre la investigación y la educación

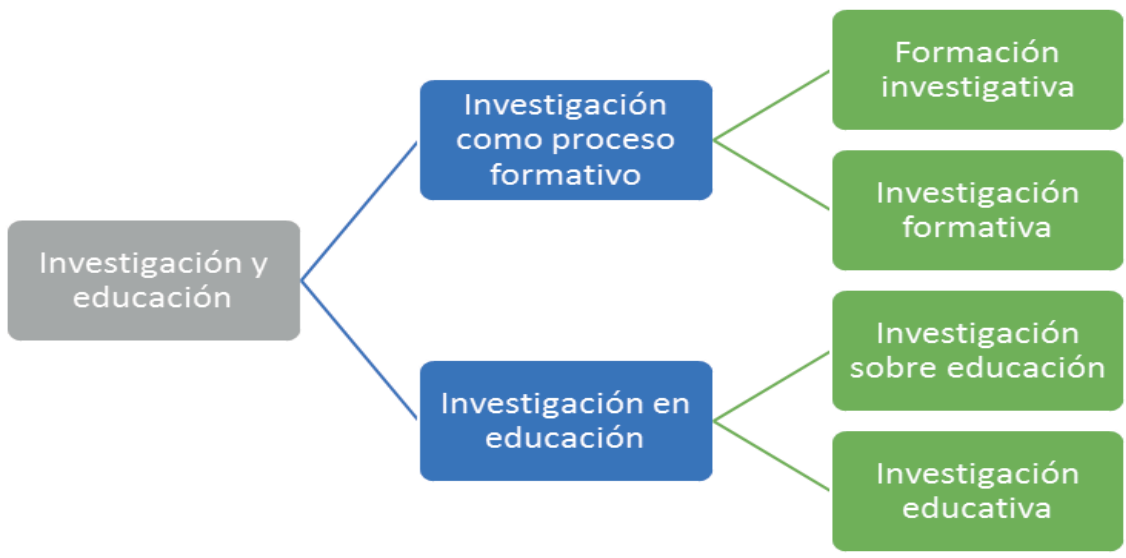

1. La investigación como proceso formativo, ubicada desde el escenario puramente educativo se subdivide en dos ramas:

1.1. La formación investigativa que se plantea como una asignatura del currículo de los programas académicos o que es desarrollada a través de semilleros de investigación, desde los que se pretende, por un lado, que el estudiante adquiera conocimientos sobre la importancia de la investigación para el desarrollo científico de una disciplina particular y para la generación de conocimiento de la misma; de igual manera se busca explotar en estos sujetos su potencial investigativo a través del desarrollo de las habilidades necesarias para identificar problemáticas y plantear soluciones directamente en el contexto social, cultural y académico en el que se encuentre.

"La formación investigativa no solo le proporciona al estudiante las herramientas necesarias para aprender a investigar, sino también una estrategia que debe atravesar todos los espacios de la formación y de la misma vida cotidiana, en lo personal, familiar y social [...]" (Marín, 2012: 21).

1.2. La investigación formativa, tiene además del desarrollo de habilidades para la investigación, que es utilizada como una estrategia didáctica para lograr niveles de pensamiento crítico-reflexivo en el estudiante, generar análisis y procesos de diálogo para lograr una comprensión adecuada de los conocimientos propios de cada asignatura. 
Así, desde el punto de vista educativo, la investigación es una herramienta supremamente valiosa en la formación de los sujetos, ya sea para desarrollar habilidades de indagación, críticas, reflexivas y analíticas, o para complementar su formación profesional y buscar la producción de conocimiento en su área de desempeño.

2. Por otro lado, la investigación rigurosa ${ }^{3}$ es desarrollada por profesionales con experiencia investigativa en cada una de las disciplinas; tiene como finalidad la obtención de conocimiento que desemboca en la producción teórica o de programas de desarrollo para generar transformación en el campo de investigación abordado.

Este tipo de investigación es la que durante siglos ha permitido la ampliación de las barreras del conocimiento, el desarrollo tecnológico, el crecimiento progresivo de teorías revolucionarias en todos los campos, pues tiene como criterio de validez la demostración empírica de sus postulados, ya sean desde el punto de vista experimental o desde diagnósticos sociales y culturales. "La investigación propiamente dicha o investigación pura es la que hace un investigador como actividad profesional con el propósito de producir conocimiento en un determinado campo del saber, mejorar, innovar un proceso o hacer un descubrimiento [...]" (Marín, 2012: 21).

Esta forma tradicional de investigación incluye el escenario educativo (Flórez, 2005), en el cual busca conocer las realidades que convergen en la escuela y el contexto que la rodea. Así, son intereses de investigación en este campo los relacionados con la transmisión del conocimiento (el aprendizaje, la enseñanza, los contenidos y la evaluación) y situaciones sociales (los comportamientos, la violencia, los valores, etc.) dentro de la escuela, como asuntos familiares (violencia intrafamiliar, valores en el hogar, etc.), económicos (trabajo, ingresos, etc.), políticos (normas, pensamiento democrático, etc.), entre muchos otros que desde el exterior del aula condicionan el buen desarrollo de los procesos educativos.

3 Con esto no se pretende insinuar que los procesos desarrollados en la investigación formativa no sean rigurosos, sino que su finalidad no es tanto la producción de conocimiento en las áreas disciplinares específicas, sino en la adquisición de habilidades para la investigación u otro tipo de competencias como ya se mencionó anteriormente. Incluso es nombrada también como "investigación propiamente dicha". 
Es sobre este escenario de investigación que se desarrollará el presente análisis, aquel que permite la transformación permanente de la educación y lo que acontece en ella, que procura su mejoramiento continuo con el fin de hacerla más pertinente con las necesidades que el contexto social exige de cada uno de los "sujetos educables" (Zambrano, 2001).

\section{Investigación en educación}

Marín (2012) se refiere a la investigación en educación, en modo amplio, como los estudios endógenos sobre la misma, las políticas, normativas, la formación docente, etc. Por su parte, Restrepo (2002) utiliza el término investigación en educación "[...] para referirse a todo estudio investigativo relacionado con educación, sea investigación educativa o investigación sobre educación. Es, por ello, genérico, los otros dos, específicos” (23).

Tal como se mencionó más arriba, la educación es un escenario dinámico que está en constante trasformación, por tanto exige de su estudio y comprensión; es en este sentido que el autor antes mencionado se aventura a una clasificación de dos tipos de investigación, ubicándola ya sea dentro del aula o fuera de ella, con el ánimo de dar reconocimiento a todas aquellas apuestas que buscan mejorarla continuamente.

En primera instancia se encuentra la investigación sobre educación, que se refiere a todos aquellos estudios que analizan el escenario educativo desde el margen, desde las fronteras disciplinares y fuera del aula. Esta es realizada por profesionales de otras disciplinas científicas que plantean opciones pedagógicas, didácticas y curriculares para que la educación sea más pertinente a los postulados teóricos o las necesidades sociales de cada contexto. "La investigación sobre educación se refiere, entonces, más a estudios científicos explicativos o comprensivos de fenómenos relacionados con la educación y que son abordados por otras ciencias y disciplinas como la filosofía, la antropología, la economía, la administración, la sociología y, por supuesto, la sicología” (Restrepo, 2002: 23).

En este marco interdisciplinar se crean departamentos encargados de estructurar teorías desde cada disciplina particular, dirigidos a estudiar la educación, así: filosofía de la educación, antropología de la educación, economía de la educación, administración educativa, sociología de la educación y sicología de la educación. 
No obstante, la investigación sobre educación ha sido la que ha realizado las propuestas sobre cómo deben desarrollarse los procesos educativos; pero es precisamente este aspecto el que plantea una gran crítica a este tipo de investigación, toda vez que la innovación y la transformación de los mismos debe partir del maestro, responsable de la asignatura, conocedor de sus estudiantes, de su contexto y de los ritmos de aprendizaje.

$\mathrm{Al}$ respecto, es posible plantear que aspectos como estos son los que han generado multiplicidad de crisis al interior de la escuela, porque los maestros parecen dedicados a replicar fórmulas ya diseñadas, apuestas generadas de "puertas para afuera", y con un gran desconocimiento de la compleja realidad que se impone al interior del aula escolar.

Precisamente, en respuesta a lo anterior, se plantea una segunda clasificación, la investigación educativa ${ }^{4}$, desde la cual se piensa en la educación de "puertas para adentro", preocupada por los procesos de aula, los comportamientos del niño, las situaciones sociales que afectan su cotidianidad escolar, es decir, por lo educativo directamente:

Por investigación educativa se entiende generalmente la centrada en lo pedagógico, sea ella referida a los estudios históricos sobre la pedagogía, a la definición de su espacio intelectual, o a la investigación aplicada a objetos pedagógicos en busca del mejoramiento de la educación, como es el caso de la indagación sobre el currículo, los métodos de enseñanza y demás factores inherentes al acto educativo (tiempo de aprendizaje, medios y materiales, organización y clima de la clase, procesos de interacción o comunicación...). Para cumplir tales propósitos, la investigación describe, clasifica, explica, predice, experimenta y controla los factores objeto de estudio, dando lugar a investigación teórica, experimental y de investigación y desarrollo de procesos y objetos educativos (Restrepo, 2002: 21).

Es en esta segunda clasificación que se halla un marco de referencia fuerte al cual apostarle y en el cual ubicar la relación entre la investigación y el escenario educativo, donde el responsable sea el maestro y el aula su escenario permanente de indagación; desde allí, se busca aportar al mejoramiento in situ de los procesos educativos y la maduración constante de los sujetos inmersos en estos: maestros y estudiantes; pues como lo manifiestan García, Villa y Toro (2009):

4 Llamada investigación en pedagogía por Marín (2012: 16). 
[...] la investigación educativa suministra al profesor elementos necesarios para reconsiderar su práctica, reconocer acontecimientos y sucesos dignos de atención y teorización, así como habilitarlo para llevar registros adecuados de cada una de las etapas de investigación e iniciar al maestro y a los estudiantes en el uso de las formas de trabajo propias de los científicos, lo cual conlleva, además, a una cualificación de su oficio (115).

\section{El rol docente: entre lo tradicional y la emergencia innovadora}

Ubicar la investigación como una de las responsabilidades del docente remite al marco de normas ${ }^{5}$ establecidas y reconocidas desde el Ministerio de Educación Nacional (MEN) de Colombia, por tanto se convierten en comprensiones propias de los contextos sociales, culturales y políticos, adjudicando al maestro el valor de formador en tanto representa intersubjetividades reconocidas y aceptadas socialmente; pero además señalan las fronteras éticas y desdibujan los significados que tradicionalmente le han sido otorgados a su propia práctica en relación con las nuevas realidades contextuales que lo afectan, provocando la transformación de su sentido, a lo que se le confiere un rol polisémico. "Hoy el docente se desempeña en una doble dimensión de su profesión: por un lado, debe ser facilitador del desarrollo del aprendizaje de sus estudiantes y, por el otro, ser un investigador de su propia práctica y de la actividad profesional y disciplinar. Ambas funciones no son excluyentes ni antagónicas; al contrario, más bien complementarias" (Marín, 2012: 11).

Por tanto no se puede desconocer la tensión que en ocasiones se presenta entre la sociedad y la práctica educativa, en atención a los vertiginosos cambios a los que se somete la sociedad contemporánea y que afectan las dinámicas del ser humano, particularmente a la comunidad estudiantil. Es allí donde la participación del docente debe consolidar acciones de apoyo en situaciones emergentes que se presentan en el aula y van más allá de lo institucional, de lo académico, y es así como se convierte en confidente, amigo, e incluso, cómplice ${ }^{6}$ de sus estudiantes; asuntos que refuerzan los

5 Ley General de Educación (115 de 1994) de la República de Colombia.

6 Mèlich (1994) al respecto de las relaciones maestro - estudiante plantea que el primero debe abandonar los distanciamientos y barreras que crea frente al segundo, para construir lazos que lo hagan cómplice del mismo y hacer del encuentro educativo un proceso de diálogo. 
vínculos al no dejar de lado el sentido de lo humano, asumiendo en primera instancia la responsabilidad social de formar sujetos aceptados socialmente y, en segundo lugar, como lo expresa Freire (1999) “[...] lo que importa es realmente ayudar al hombre a recuperarse. [...] hacerlos agentes de su propia recuperación. Es ponerlos en una posición conscientemente crítica frente a sus problemas [...]" (50), generar su transformación crítica emancipatoria, de participación como asunto de libertad personal.

En este sentido, la responsabilidad del docente no solo se transfiere al campo de la atención individual del estudiante, sus acciones involucran el compromiso con la comunidad educativa, palabras que hacen eco a lo expresado por Freire (1999) "[...] toda comprensión corresponde entonces tarde o temprano a una acción. Luego de captado un desafío, comprendido, admitidas las respuestas hipotéticas, el hombre actúa. La naturaleza de la acción corresponde a la naturaleza de la comprensión [...]" asunto que exige una labor organizacional en la gestión de su práctica educativa donde se involucran componentes esenciales en el proceso de enseñanza y aprendizaje, como la pedagogía, la didáctica, el currículo y la evaluación educativa.

En esta línea de pensamiento, la pedagogía vista como un saber que se preocupa por la formación del ser humano en todas sus dimensiones (ser, saber y hacer), debe asumir con claridad el concepto de hombre/ mujer (persona), tipo de conocimiento y, por tanto, tipo de sociedad a la que espera aportar. Por ello, cuando el maestro reflexiona sobre lo pedagógico justifica su práctica, permeada por las necesidades educativas de los estudiantes y su contexto.

En este sentido, la pedagogía expone y analiza los principios y los fines que deben guiar su labor, sin los cuales ésta no sería más que un ejercicio irreflexivo y sin un horizonte hacia el cual dirigir sus esfuerzos.

Esfuerzos mediados por la didáctica, sin la cual la pedagogía no sería más que un ideal distante de la realidad y de los verdaderos alcances de la educación; en palabras de Zambrano (2005) "La didáctica crea situaciones experimentales, organiza instrumentos de observación, reflexiona sobre los aprendizajes y algunas prácticas contractuales de la enseñanza, le explica a las otras ciencias sus avances y les facilita una mayor comprensión del hecho escolar [...]" (57). Comprensión que se logra mediante la 
organización del conocimiento, de aquellos saberes que son pertinentes para ser aprendidos por el estudiante, fijados en las propuestas y prácticas curriculares institucionales con la pretensión de abordar los problemas situacionales del aula, hechos o fenómenos que a la luz del paradigma interpretativo se pueden traducir en espacios de deliberación, de actividad dialógica, de crítica, de propuestas, consensos, escenarios que invitan a la exploración de la investigación reflexiva en el aula.

Saberes en interacción con la comprensión de la realidad, el sentido y la intencionalidad que tiene el docente en su discurso temático y que es necesario valorar; más allá de la mirada técnica-racional se puede decir que la evaluación entra a jugar un importante papel en el proceso de análisis, reflexión y acción en el aprendizaje significativo y como dispositivo de renovación del hecho educativo.

Lo expuesto propone un análisis integral de los elementos que por diversas situaciones han sido aislados unos de otros, y pocas veces se abordan como un conjunto armónico de la misma maquinaria, en donde la ausencia de alguno sería un traspié en el acertado desarrollo de una educación transformadora

\section{Reflexión y acción del hecho educativo: Una propuesta para dar significado a la investigación en el aula}

Como se expone en líneas anteriores, a la educación transformadora desde una perspectiva crítica se le adjudica la transmisión reflexiva del conocimiento, y es la investigación la llamada a producirlo en el acercamiento inquieto del investigador a la realidad, quien se pregunta constantemente sobre el mundo que aparece ante él. Para ello, estas expectativas requieren ser planeadas, centrar los esfuerzos hacia el logro de un fin y la resolución de la problemática formulada. Al respecto Tamayo (1999) menciona que "La investigación es un proceso que, mediante la aplicación del método científico, procura obtener información relevante y fidedigna, para entender, verificar, corregir o aplicar el conocimiento" (34).

La investigación científica brinda apertura al cuestionamiento, a la recreación e innovación constante del sujeto inmerso en fenómenos sociales y naturales, en disposición de comprenderlos, explicarlos y transformarlos para beneficio de la comunidad. Asunto que implica la definición de un 
interés investigativo, traducido en la formulación de un cuestionamiento, objetivos realizables que conlleven a la explicación o comprensión de dicho problema, elementos teóricos que fundamenten la pertinencia y claridad sobre el tema, así como la metodología o recorrido que lleve al investigador a alcanzar los objetivos (Zuluaga, et ál., 2003: 73-109).

El paradigma crítico exige tanto de la investigación como de la educación, la transformación en las relaciones, dialogicidad, la mirada atenta a realidades, a problemáticas emergentes, posibilitando la fusión de investigación y educación para renovar el re-encuentro entre maestros y estudiantes, es así como "[...] la teoría crítica de la educación, [...] asume la dialéctica como su metodología. El profesor se ubica como un investigador colaborativo" (Galeano, 2009: 87).

No obstante, cuando se pide a los docentes que incorporen la investigación a su práctica se genera apatía al no contar con los espacios, tiempos y recursos necesarios para hacer evidente la dinámica en el aula y la diversidad de situaciones que en ella se configuran; para el maestro implica sumar tareas a las que ya debe cumplir en la institución educativa. Así, quienes investigan lo hacen como responsabilidades académicas que se convierten en exigencias para la obtención de un título (de postgrado) para ascender en el escalafón, y otros lo hacen por motivación personal.

Frente a lo anterior Jaramillo (s.f.) se pregunta, ¿cómo hacer de la investigación procesos que desbordan la razón? Cuestión ante la cual expone que:

Tal vez si nos constituimos con aquello que nos desborda, podremos sentirla como oportunidad para ensanchar horizontes de vida y no seguir atrapados en su instrumentalización. Es hacer de la investigación un jugar para que se vuelva lúdica y significado; enamorarnos del área o tema que nos apasiona para sentir gozo en su exploración. Todo ello, con la sospecha de que nos faltó algo más por descubrir, por encontrar, otra muralla que franquear: un deseo inacabado (9).

A partir de esta fundamentación se lanza una propuesta que integre los elementos propios del acto educativo con los de investigación y que permita al docente indagar su experiencia inmediata en el aula, pensar la preparación de la clase como construcción de un proyecto investigativo y que su práctica se convierta en escenario de indagación. 
Para esto fue necesario comprender que tanto el proyecto de investigación como un plan curso se preparan desde cinco principios fundamentales, a saber: ontológico, teleológico, epistemológico, metodológico y axiológico, tal como se expone en la ilustración 2.

Ilustración 2. Principios fundamentales de la investigación y la educación INVESTIGACIÓN

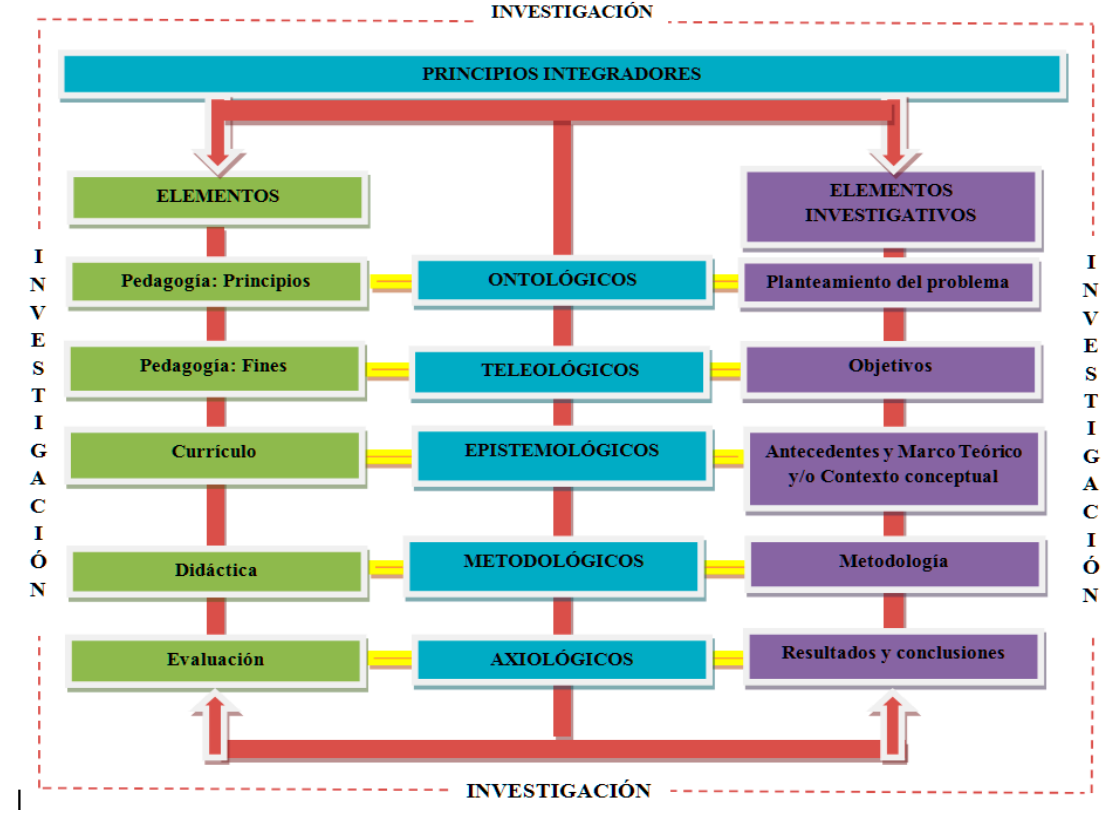

a) El principio ontológico entendido como el ser de las cosas que existen alrededor del sujeto, su razón de ser y de existir. Tanto para la educación como para la investigación se refiere al principio que guía los esfuerzos del maestro investigador; se construye a partir del análisis del contexto y sus necesidades particulares.

En este sentido, lo ontológico se construye a partir del análisis reflexivo, atento y curioso sobre el escenario socio-cultural, justo allí donde encuentra la justificación para el ejercicio educativo e investigativo.

b) El principio teleológico se encarga de pensar los fines de la investigación y la educación, el horizonte hacia donde se dirigen los esfuerzos en la formación del sujeto y al alcance del conocimiento. Por ello, se sugiere, que se elabore en doble sentido: el proceso formativo del estudiante y la construcción permanente del conocimiento. 
c) La organización propia de los conocimientos la ubicamos en el principio epistemológico fundamentado en la teoría; permite dar claridad a los elementos tanto de la investigación como del acto educativo.

d) Los tránsitos previstos como trayectos de investigación y educación los asignamos al principio metodológico como una de las rutas posibles que el docente-investigador planea en términos de estrategias e instrumentos, necesarios para recorrer el camino. Es la metodología a través de la cual se alcanzan los objetivos previstos, los aprendizajes (mediante la didáctica) y la construcción de nuevos saberes (el método investigativo).

e) Para llegar a un todo comprensible del proceso educativoinvestigativo no se puede desligar lo axiológico como principio vital y valorativo del papel del sujeto y las estrategias utilizadas para el alcance de los objetivos propuestos. Por tanto, nos exige estar atentos a problemáticas que se desprendan de este análisis y finalmente retroalimentar todo el trayecto recorrido.

\section{A modo de cierre}

La investigación guarda en su ejercicio un alto potencial transformador tal como es requerido por las actuales necesidades del mundo contemporáneo, dominado por el auge de las tecnologías y la influencia de los medios de comunicación.

Así, cuando se vincula la investigación al ejercicio docente, este se convierte en investigador y la educación sufre una transformación positiva, pues permite al maestro identificar problemáticas del contexto educativo, posibilidades de transformación, y renovar permanentemente su labor.

Pensar la pedagogía, la didáctica, el currículo y la evaluación educativa en una relación directa con los elementos ontológicos, epistemológicos y metodológicos de la investigación, se presenta como novedad que deja entrever una nueva posibilidad de alcanzar las transformaciones mencionadas y que la labor docente no se realice distante de la labor investigativa al ser apropiada en el marco del ejercicio docente. 
Así mismo se expone la investigación como compromiso educativo y necesario en la actualización de conocimientos, en relación bidireccional maestro-estudiante, condicionado por un contexto y, desde lo que plantea el paradigma crítico, es necesario propiciar herramientas para comprenderlo, analizarlo y buscar su transformación permanente como un ser en emancipación hacia su libertad.

\section{Referencias}

Bedoya M., J. I. (2005). Epistemología y pedagogía [6 $6^{a}$ ed.] Bogotá: Ecoe Ediciones.

BOTERo U., D. (s.f.). La utopia: una posibilidad para asumir creativamente un proceso de investigación. [Documento inédito].

Deslauriers, J. P. (2004). Investigación cualitativa. Pereira, Colombia: Editorial Papiro. Recoger

Dos Santos Filho, J. C. \& SÁnchez G., S. A. (1997). Investigación educativa. Cantidad - Cualidad. Bogotá: Cooperativa Editorial Magisterio.

Flórez O., R. (2005). Pedagogía del conocimiento [2a ed.] Bogotá: McGraw-Hill Interamericana.

FreIRE, P. (1999). La educación como práctica de la libertad [48ª ed.]. España: Siglo XXI Editores.

Hernández S., R.; Fernández C., C. \& Baptista L., P. (2010). Metodología de la investigación [ $5^{a}$ ed.] México, D.F.: McGraw-Hill - Interamericana Editores, S. A.

Hernández, C. A. \& López C., J. (2002). Disciplinas. Bogotá: ICFES, Secretaría General - Procesos Editoriales.

Hurtado De Barrera, J. (2000). El proyecto de investigación. Caracas: Fundación Sypal.

Jaramillo E., L. G. (s.f.). Amor y juego - Investigación y deseo. [Documento inédito].

(2012). 'Deshilachando sobre suelo resbaladizo'. Revista Nómadas [37]. Universidad Central de Colombia.

Marín G., J. D. (2012). La investigación en educación y pedagogía. Bogotá: Universidad Santo Tomás, Departamento de Publicaciones.

Mèlich, J. C. (1994). Del extraño al cómplice: La educación en la vida cotidiana. Barcelona: Anthropos.

Murcia P., N. \& Jaramillo E., L. G. (2001). Seis experiencias en investigación cualitativa. Armenia, Colombia: Editorial Kinesis. 
(2008). Investigación cualitativa "La complementariedad". Armenia, Colombia: Editorial Kinesis.

PIÑA, J. M. (2010). El cristal con que se mira. Diversas perspectivas metodológicas en educación. México: Universidad Nacional Autónoma de México [Posgrado en Pedagogía] - Ediciones Díaz Santos S.A.

Schutz, A. (2008). El problema de la realidad social [2 $2^{\mathrm{a}}$ ed.] Buenos Aires: Amorrortu Editores.

Restrepo G., B. (2002). 'Investigación en educación'. Programa de especialización en métodos y técnicas en investigación social [Módulo 7].

Tamayo y Tamayo, M. (1999). 'La investigación'. Serie aprende a investigar. [Módulo 2]. Bogotá: ICFES.

García M., O. J.; Villa Pineda, C. A. \& Toro González, L. E. (2009). ‘Sentido que dan docentes y estudiantes a la investigación en los programas de licenciatura de la Universidad de Caldas'. Revista Latinoamericana de Estudios Educativos [5, 2, 111 - 139]. Manizales, Colombia: Editorial Universidad de Caldas.

Zambrano L., A. (2005). Didáctica, pedagogía y saber. Bogotá: Cooperativa Editorial Magisterio.

(2001). La mirada del sujeto educable: la pedagogía y la cuestión del otro. Santiago de Cali: Grupo Editorial Nueva Biblioteca Pedagógica.

Zuluaga, O. L.; Echeverry, A.; Martínez, A.; Quiceno, H.; Sáenz, J. \& Álvvarez, A. (2003). Pedagogía y epistemología. Bogotá: Cooperativa Editorial Magisterio. 